\title{
Clinical spectrum of severe chronic central serous chorioretinopathy and outcome of photodynamic therapy
}

This article was published in the following Dove Press journal:

Clinical Ophthalmology

\author{
Danial Mohabati ${ }^{1,2}$ \\ Elon HC van Dijk' \\ Thomas J van Rijssen' \\ Eiko K de Jong ${ }^{3}$ \\ Myrte B Breukink ${ }^{3}$ \\ Jose P Martinez-Ciriano ${ }^{4}$ \\ Greet Dijkman' \\ Carel B Hoyng ${ }^{3}$ \\ Sascha Fauser ${ }^{5}$ \\ Suzanne Yzer ${ }^{4}$ \\ Camiel JF Boon ${ }^{1,6}$ \\ 'Department of Ophthalmology, \\ Leiden University Medical Center, \\ Leiden, the Netherlands; ${ }^{2}$ Department \\ of Ophthalmology, Rotterdam \\ Ophthalmic Institute, Rotterdam, \\ the Netherlands; ${ }^{3}$ Department of \\ Ophthalmology, Donders Institute \\ for Brain, Cognition and Behaviour, \\ Radboud University Medical \\ Center, Nijmegen, the Netherlands; \\ ${ }^{4}$ Department of Ophthalmology, The \\ Rotterdam Eye Hospital, Rotterdam, \\ the Netherlands; ${ }^{5}$ Department of \\ Ophthalmology, University Hospital \\ of Cologne, Cologne, Germany; \\ ${ }^{6}$ Department of Ophthalmology, \\ Academic Medical Center, University \\ of Amsterdam, Amsterdam, \\ the Netherlands
}

Correspondence: Camiel JF Boon Department of Ophthalmology, Leiden University Medical Center, Albinusdreef 2, 2333 ZA Leiden, the Netherlands $\mathrm{Tel}+3$ I 7| 5265936

Fax +3। 7| 5248222

Email c.j.f.boon@lumc.nl
Purpose: To describe a spectrum of severe chronic central serous chorioretinopathy (cCSC) cases and their response to photodynamic therapy (PDT).

Patients and methods: A total of 66 patients ( 81 eyes) with active severe cCSC were studied, and their response to PDT was compared with a control group consisting of 35 active cCSCs (37 eyes) that did not display characteristics of severity. Best-corrected visual acuity (BCVA) and complete resolution of subretinal fluid (SRF) were considered as main outcome measures.

Results: In severe cCSC cases, we found cumulative areas of diffuse atrophic retinal pigment epithelium alterations in 48 eyes (59\%), multiple "hot spots" of leakage in 36 eyes (44\%), posterior cystoid retinal degeneration in 25 eyes (31\%), and 13 eyes (16\%) had a diffuse leakage on fluorescein angiography. After PDT treatment, BCVA increased in both groups, from 66 to 72 Early Treatment of Diabetic Retinopathy Study (ETDRS) letters in the case group $(P<0.001)$, and from 78 to 82 ETDRS letters in the control group $(P<0.001)$. SRF had resolved completely in $87 \%$ of severe cCSC cases and $95 \%$ of controls at final follow-up visit.

Conclusion: A spectrum of severe cCSC exists, and PDT seems to be an effective treatment in both severe cCSC and nonsevere cCSC in terms of resolution of SRF. Final BCVA shows a significant improvement in both groups after PDT treatment.

Keywords: chronic central serous chorioretinopathy, photodynamic therapy, posterior cystoid retinal degeneration, severe phenotype, therapeutic outcome

\section{Introduction}

Central serous chorioretinopathy (CSC) is characterized by an accumulation of serous fluid under the retina, often affecting the macula. ${ }^{1}$ The presence of subretinal fluid (SRF) is presumed to result from a dysfunctional choroid that is swollen and hyperpermeable, in association with a disturbance of the retinal pigment epithelium (RPE) that compromises the outer blood-retinal barrier. ${ }^{2,3}$ SRF accumulation causes a neuroretinal detachment with resulting photoreceptor dysfunction and vision loss. ${ }^{4-6}$ Other common abnormalities in CSC include RPE detachments, a variable degree in RPE atrophy, focal leakage ("hot spots"), or diffuse areas of fluorescein leakage through an RPE defect visualized with fluorescein angiography (FA), ${ }^{2,3}$ Indocyanine green angiography (ICGA) often shows more widespread choroidal abnormalities compared with those on FA, with leakage from a hyperpermeable, thickened, and congested choroid. ${ }^{7-9}$ The use of corticosteroids, endogenous hypercortisolism, mental stress, and pregnancy have been suggested to be risk factors for the development of CSC. ${ }^{10,11}$ In addition, genetic protective and risk factors have been shown to be associated with CSC. ${ }^{12-15}$ Two main subtypes of CSC are often distinguished. Patients with 
acute CSC (aCSC) typically present with sudden vision loss due to a focal leak in the RPE, without significant atrophic RPE changes, and show spontaneous resolution within weeks. ${ }^{6,7}$ Compared with this aCSC phenotype, patients with chronic CSC (cCSC) tend to have prolonged subfoveal SRF accumulation, more atrophic RPE changes, and more diffuse and/or multifocal leakage on FA and ICGA. In contrast to the relatively favorable prognosis of aCSC, cCSC is typically not self-limiting and is associated with progressive visual loss and a decreased vision-related quality of life. ${ }^{5}$ Therefore, treatment is generally advocated in cCSC. ${ }^{16}$ Previously, multiple treatments have been suggested, including conventional thermal laser, subthreshold micropulse diode laser, photodynamic therapy (PDT), anti-vascular endothelial growth factor injection, and oral mineralocorticoid receptor antagonist agents. ${ }^{7}$ The PLACE trial, the first large prospective randomized controlled trial for cCSC, has shown that PDT with reduced settings seems to be the superior treatment option in terms of efficacy and safety. ${ }^{17}$ The extent of retinal abnormalities may vary strongly between cCSC cases. A significant subgroup of cCSC patients seems more severely affected and displays relatively large areas of RPE atrophy, multifocal areas of leakage on FA, posterior cystoid retinal degeneration (PCRD), and/or subretinal fibrin accumulation. ${ }^{18-21}$ Despite the clinical observation that cCSC includes a spectrum of severe disease characteristics, ${ }^{22}$ little is known about this clinical spectrum, its long-term visual prognosis, and treatment outcome in this subgroup. In this study, we hypothesized that those cCSC cases with the most severe phenotype may have a different disease course and a less favorable treatment outcome. Here, we describe the clinical spectrum and outcome of PDT in a large group of cCSC patients with a severe clinical phenotype, and compare them with a cohort of cCSC cases that do not manifest those characteristics of presumed severity.

\section{Methods}

\section{Patients}

Approval for this study was obtained at the local institutional review boards in all participating centers, and the study adhered to the tenets of the Declaration of Helsinki. The cCSC patients included in this study were diagnosed and treated at the Department of Ophthalmology of Leiden University Medical Center (Leiden, the Netherlands), and the Rotterdam Eye Hospital (Rotterdam, the Netherlands). The diagnosis of cCSC was defined as the presence of cCSC-related visual symptoms for $>6$ months, as well as the presence of chronic SRF on optical coherence tomography (OCT) for $>3$ months, RPE window defects on FA with at least 1 "hot spot" and/or diffuse leakage, and corresponding hyperfluorescent areas on ICGA when available. cCSC patients were subsequently categorized as having a severe phenotype (cases) or a nonsevere phenotype (controls).

\section{Criteria of severity of cCSC}

A cCSC phenotype was considered severe when at least one of the following clinical findings was observed at some point during the disease course: 1$)$ cumulative areas ( $>5$ optic disc diameters) of diffuse atrophic RPE alterations (DARA) as visualized on mid-phase FA (previously described as: diffuse retinal pigment epitheliopathy), ${ }^{20,23} 2$ ) at least 2 "hot spots" of leakage separated by at least 1 disc diameter of nonhyperfluorescent healthy-appearing retina on mid-phase FA (multifocal "hot spots"); 3) an area of diffuse fluorescein leakage $>1$ optic disc diameter on mid-phase FA, without an evident leaking focus (diffuse leakage); and 4) presence of PCRD assessed on OCT, as described previously. ${ }^{18,19}$ The described abnormalities were considered relevant only when manifesting within the largest outer temporal vascular arcades. Patients were categorized as having a nonsevere cCSC (controls) when none of the previously mentioned criteria of severity where present. We excluded patients with evidence of other retinal diagnoses, such as a history of exudative age-related macular degeneration, suspicion of secondary choroidal neovascularization, polypoidal choroidal vasculopathy, multifocal choroiditis, retinal vascular occlusions, pseudoxanthoma elasticum, amblyopia, and severe myopia (more than -6 diopters).

\section{Clinical examinations}

All patients underwent an extensive ophthalmological evaluation and multimodal imaging at diagnosis and during follow-up: Early Treatment of Diabetic Retinopathy Study (ETDRS) best-corrected visual acuity (BCVA) measurement (a previously published method was used to convert Snellen BCVA to ETDRS BCVA, when this was not available), ${ }^{24}$ slit-lamp examination, and/or color fundus photography (Topcon Corp; Tokyo, Japan, or Carl Zeiss Meditec; Dublin, CA, USA), either time-domain OCT (Cirrus HD-OCT; Carl Zeiss Meditec, or OCT-HS100; Canon Inc, Tokyo, Japan) or spectral-domain OCT (Spectralis HRA+OCT; Heidelberg Engineering, Heidelberg, Germany), FA (Topcon Corp or Spectralis HRA+OCT, or Carl Zeiss Meditec), and ICGA (Topcon Corp or Spectralis HRA+OCT, or Carl Zeiss Meditec). 


\section{Photodynamic therapy}

In this study, PDT was performed with different reduced settings, based on the preference of the treating ophthalmologist. These settings were as follows: half-dose (3 $\mathrm{mg} / \mathrm{m}^{2}$ verteporfin [Visudyne ${ }^{\circledR}$, Novartis international AG, Basel, Switzerland]), or half-time (a treatment duration of 42 seconds), or half-fluence $\left(25 \mathrm{~J} / \mathrm{cm}^{2}\right)$ compared with the original PDT settings described for neovascular age-related macular degeneration. PDT treatment was performed with a standard PDT laser wavelength of $689 \mathrm{~nm}$. All subjects in this study were treated when there was no spontaneous resolution of SRF. Here, we studied the effect of the first (initial) PDT after manifestation of criteria of severity in the case group and the first (initial) PDT after diagnosis of cCSC in the control group.

\section{Definition of clinical outcome measures}

The primary outcome measures included post-PDT BCVA and final BCVA, as well as complete resolution of SRF as observed on OCT. For a longitudinal overview of disease progression, relevant outcome measures were monitored at different moments throughout follow-up: at disease diagnosis, prior to the first PDT treatment, at the first visit after PDT treatment, and at final available follow-up visit. Information regarding the use of steroid-containing medication (through all possible modes of administration) and endogenous hypercortisolism was collected.

\section{Statistical analysis}

Statistical analysis was performed using IBM SPSS software for Windows, version 23 (IBM Corp, Armonk, NY, USA). Either a paired samples $t$-test or an unpaired $t$-test was used for continuous numerical data. Categorical data were analyzed using a chi-squared test. A survival analysis was performed and a Kaplan-Meier survival plot was generated comparing cases with controls in terms of resolution of SRF. The event was used as follows: the moment of complete resolution of SRF on OCT after initial PDT. A log-rank test was used to compare the period of time until this event was first documented among cases and controls. A univariate analysis was performed using Pearson's correlation to evaluate the characteristics that associated with final visual outcome. A value of $P<0.05$ was considered significant in all performed tests.

\section{Ethics approval}

All procedures performed in this study involving human participants were in accordance with the ethical standards of the institutional and/or national research committee and the 1964 Helsinki Declaration and its later amendments or comparable ethical standards. The local institutional review boards in all participating centers ("Medisch Ethische Toetsingscommissie" [METC] in Leiden University Medical Center, The METC in the Radboud University Medical Center, and the "Wetenschapscommissie" in the Rotterdam Eye Hospital) did not require written consent from the participants for reviewing their medical records, as all data were anonymized upon collection.

\section{Results}

\section{Patient characteristics}

After retrospectively reviewing medical records of $650 \mathrm{CSC}$ patients from the participating centers, $66 \mathrm{cCSC}$ patients (81 eyes) were included in the case group who met the criteria of severity, and $35 \mathrm{cCSC}$ patients (37 eyes) were included in the control group who did not exhibit any of the aforementioned severity criteria. An overview of patient characteristics is provided in Table 1 . The distribution of the criteria of severity was as follows: 48 eyes (59\%) showed DARA, 36 eyes (44\%) showed multifocal "hot spots" (range: 2-6 "hot spots"), 25 eyes (31\%) showed PCRD, and 13 eyes $(16 \%)$ showed diffuse leakage on FA. Most cases (50 eyes, $62 \%$ ) manifested multiple criteria of severity (Figure 1). All cases and controls were treated with PDT. However, only 63 eyes $(78 \%)$ in the case group were treatment-naive before the studied PDT was performed (Table 2). Moreover, 25 eyes

Table I Demographic characteristics in severe chronic CSC patients (cases) and nonsevere chronic CSC patients (controls)

\begin{tabular}{llll}
\hline Characteristics & Cases & Controls & P-value \\
\hline Patients (eyes) & $66(8 I)$ & $35(37)$ & $\mathrm{n} / \mathrm{a}$ \\
Male, $\mathrm{n}(\%)$ & $69(85)$ & $28(76)$ & 0.210 \\
Caucasian ethnicity, $\mathrm{n}(\%)$ & $72(89)$ & $27(73)$ & $\mathbf{0 . 0 2 9}$ \\
Mean age at diagnosis in years (range) & $49(29-78)$ & $47(30-72)$ & 0.243 \\
Mean time from CSC diagnosis to final visit in years (range) & $6(0-30)$ & $2(0-7)$ & $<\mathbf{0 . 0 0 1}$ \\
Subjects with recent use of steroids, ${ }^{2} \mathrm{n}(\%)$ & $17^{\mathrm{b}}(2 \mathrm{l})$ & $13(35)$ & 0.110 \\
\hline
\end{tabular}

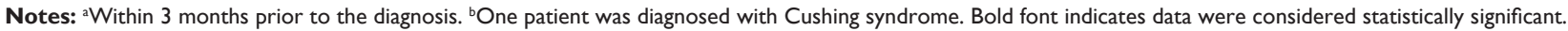
Abbreviations: BCVA, best-corrected visual acuity; CSC, central serous chorioretinopathy; n/a, not applicable. 

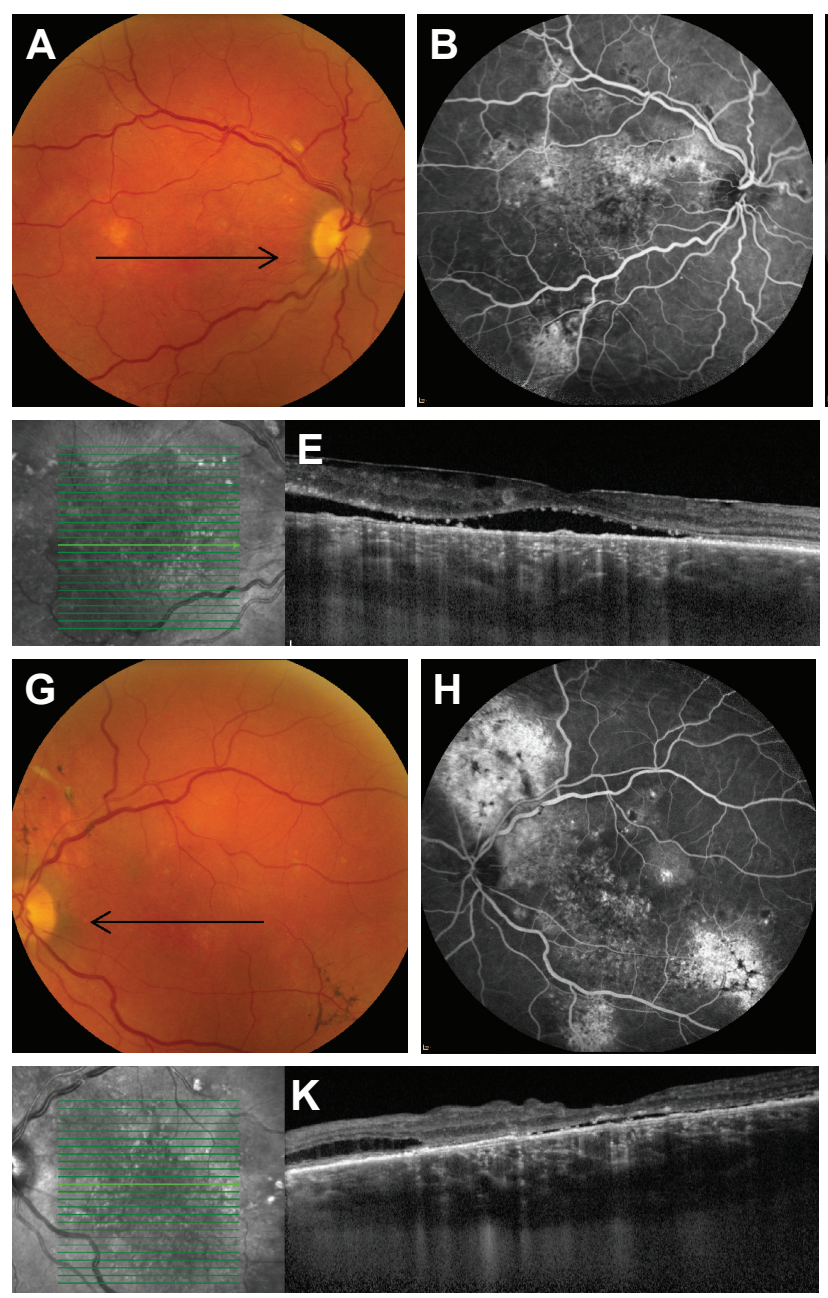
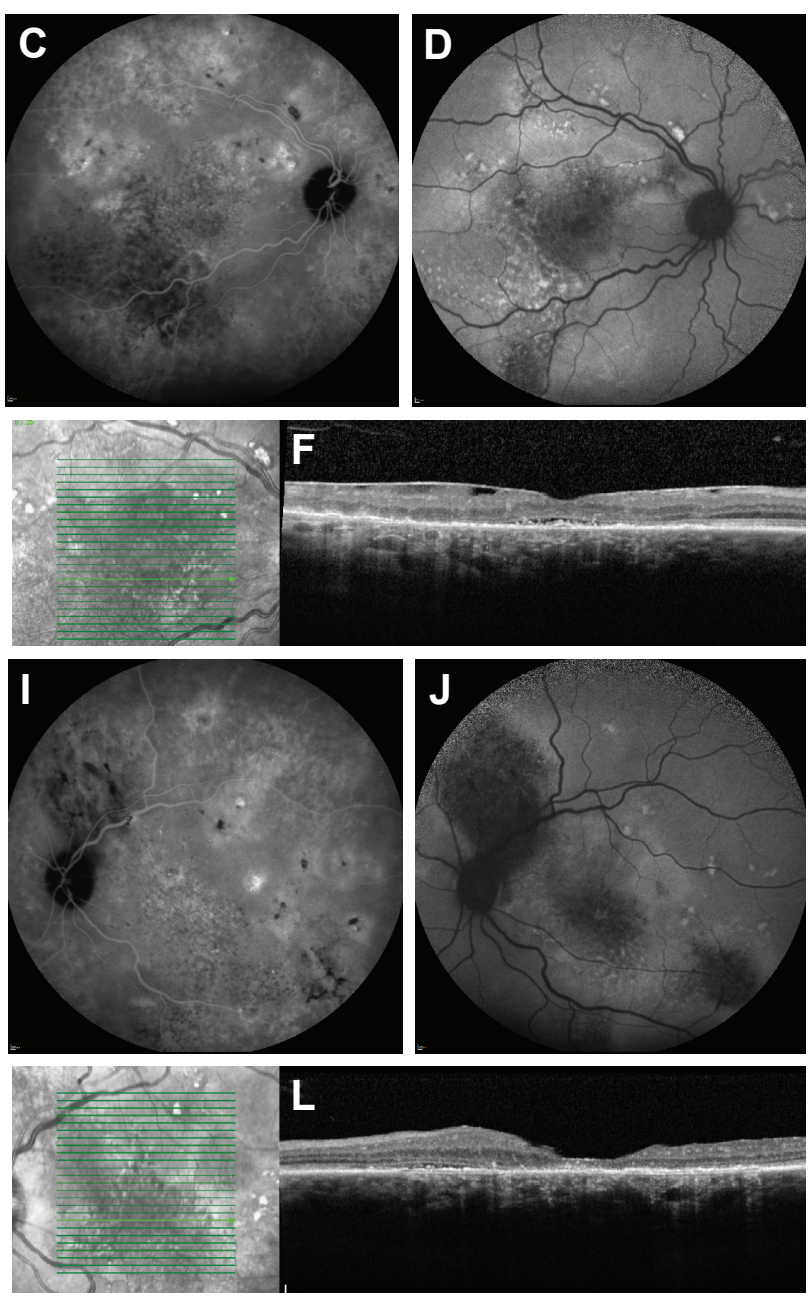
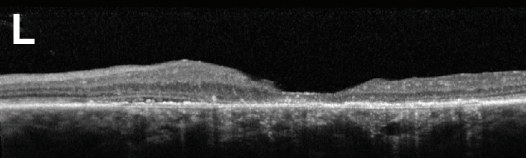

Figure I Multimodal imaging of a 7I-year-old male patient with severe bilateral chronic central serous chorioretinopathy (A-F: right eye, G-L: left eye).

Notes: On color fundus photography, atrophic RPE alterations were seen in the inferotemporal quadrant of the left eye (G). Multifocal "hot spots" of leakage (H) and extensive areas of atrophy were seen on fluorescein angiography (B, H). Fundus autofluorescence showed large areas of hypo- and hyper-autofluorescent abnormalities corresponding to the RPE changes, extending to outside the macula (D, J). Indocyanine green angiography images (C, I) showed multifocal areas of diffuse choroidal abnormalities and leakage. OCT revealed in both eyes epiretinal membrane and subretinal fluid centrally in the macula (E, K). Posterior cystoid retinal degeneration was seen in the outer nuclear layer of the nasal macula of the left eye (K). Ten weeks after half-dose photodynamic therapy, which was only performed in the left eye, both subretinal fluid and posterior cystoid retinal degeneration had disappeared (L). The black arrows on the color fundus photography images correspond to the scanning plane on the OCT scans (E, F, K, L).

Abbreviations: OCT, optical coherence tomography; RPE, retinal pigment epithelial.

Table 2 Treatment specifications in severe chronic CSC (cases) and nonsevere chronic CSC (controls)

\begin{tabular}{|c|c|c|c|c|}
\hline PDT & Treatment characteristics & Cases & Controls & $P$-value \\
\hline \multirow{5}{*}{$\begin{array}{l}\text { Before study PDT } \\
\text { treatment }\end{array}$} & Treatment-naive eyes (\%) & $63(78)$ & $37(100)$ & 0.002 \\
\hline & Eyes with previous treatments (\%) & 9 (II) conventional thermal laser & $\mathrm{n} / \mathrm{a}$ & $\mathrm{n} / \mathrm{a}$ \\
\hline & & 4 (5) anti-VEGF injections $\mathrm{s}^{\mathrm{a}}$ & & \\
\hline & & I (I) PDT & & \\
\hline & & 2 (2) multiple treatments & & \\
\hline Regarding study & PDT settings (\%) & $72(89)$ half-dose & 31 (84) half-dose & 0.454 \\
\hline \multirow[t]{3}{*}{ PDT treatment } & & 7 (9) half-time & 5 (I3) half-time & \\
\hline & & 2 (2) half-fluence & I (3) unknown & \\
\hline & $\begin{array}{l}\text { Median time from diagnosis until } \\
\text { the study PDT in weeks (range) }\end{array}$ & $5(0-234)$ & $31(3-354)$ & $<0.001$ \\
\hline
\end{tabular}

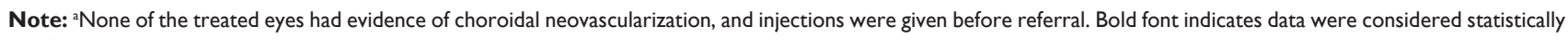
significant.

Abbreviations: CSC, central serous chorioretinopathy; n/a, not applicable; PDT, photodynamic therapy; VEGF, vascular endothelial growth factor. 
Table 3 Additional treatments after initial PDT in severe chronic CSC (cases) and nonsevere chronic CSC (controls)

\begin{tabular}{lll}
\hline Treatment characteristics & Cases & Controls \\
\hline Total number of eyes that received additional treatments (\%) & $25^{\mathrm{a}}(3 \mathrm{I})$ & $6^{\mathrm{a}}(7)$ \\
Number of eyes that received additional PDT treatments (\%) & $25^{\mathrm{b}}(100)$ & $6^{\mathrm{b}}(100)$ \\
Number of eyes that received additional conventional laser (\%) & I (4) & $0(0)$ \\
Reason for additional treatment (\%) & II (44) recurrence of SRF & I (I7) recurrence of SRF \\
& I4 (56) insufficient response to PDT & 5 (83) insufficient response to PDT
\end{tabular}

Notes: a'Some eyes received more than one type of additional treatment. ${ }^{\circ}$ Range of extra PDTs needed in cases: I-2, and in controls: I.

Abbreviations: CSC, central serous chorioretinopathy; PDT, photodynamic therapy; SRF, subretinal fluid.

among the cases (31\%), and 6 eyes among the controls $(16 \%)$ received additional treatments after initial PDT and before the final visit (Table 3).

\section{Best-corrected visual acuity}

Baseline BCVA before initial PDT was $66 \pm 19$ ETDRS letters in cases, which was significantly lower compared with controls with $78 \pm 11$ ETDRS letters $(P=0.001)$. After initial PDT, BCVA increased significantly compared with the baseline to $72 \pm 18$ and $82 \pm 9$ ETDRS letters in cases $(P<0.001)$ and controls ( $P=0.002)$, respectively, at first control visit (on average 7 weeks after initial PDT). The final available BCVA (on average 90 weeks after initial PDT) was $72 \pm 21$ and $82 \pm 11$ ETDRS letters in the case and control groups, respectively. Patients in the case group had a significantly lower BCVA at the final follow-up visit compared with the control one $(P=0.001)$ (Table 4). A Pearson correlation analysis demonstrated that a statistically significant positive correlation existed between final BCVA outcome and the baseline BCVA before PDT ( $r=0.8, P<0.001$ ). Other characteristics, such as age, gender, Caucasian ethnicity, and the mean time from CSC diagnosis to final visit did not show any significant correlation with the final BCVA outcome.

\section{Resolution of subretinal and intraretinal fluid}

At the first visit after initial PDT, a complete SRF resolution was achieved in 56 eyes (70\%) among cases and 28 eyes (78\%) among controls $(P=0.386)$. At final visit, SRF had resolved in 71 eyes (88\%) among cases, and 35 eyes (95\%) among controls $(P=0.247)$. When comparing the treatmentnaive severe cases with the treatment-naive controls, a similar pattern was observed (Table 4). A survival analysis (event was defined as complete resolution of SRF) showed a moderate trend for subjects in the control group to attain a complete resolution of SRF faster (Figure 2). The estimated median duration to achieve complete resolution of SRF after treatment initiation in cases was 8 weeks $(95 \%$ CI: 7-9), compared with 7 weeks in controls (95\% CI: $7-8[P=0.281])$. In the case group, PCRD was observed in 25 eyes (31\%). A complete resolution of PCRD after initial PDT was observed in 11 of these eyes (44\%) at first visit (Figure 3). In 12 eyes (48\%), PCRD was clearly reduced but not absent, and in 2 eyes ( $8 \%$ ), PCRD remained unchanged after initial PDT. At final visit, on average after 96 weeks of follow-up, 13 eyes (52\%) had persistent PCRD after previous PDT treatment.

Table 4 Clinical outcome after PDT with reduced settings in severe chronic CSC (cases) and nonsevere chronic CSC (controls)

\begin{tabular}{|c|c|c|c|}
\hline Clinical outcome measures & Cases & Controls & $P$-value \\
\hline BCVA before initial PDT in ETDRS letters \pm SD & $66 \pm 19$ & $78 \pm 11$ & $<0.001$ \\
\hline BCVA at first follow-up in ETDRS letters \pm SD & $72 \pm 18$ & $82 \pm 9$ & $<0.001$ \\
\hline$B C V A$ at final follow-up in ETDRS letters $\pm S D$ & $72 \pm 21$ & $82 \pm 11$ & 0.001 \\
\hline Resolution of SRF at first follow-up, ${ }^{a} n(\%)$ & $56(70)$ & $28(78)$ & 0.386 \\
\hline Resolution of SRF at final follow-up, ${ }^{b} \mathrm{n}(\%)$ & $71(88)$ & $35(95)$ & 0.247 \\
\hline Resolution of SRF in treatment-naive eyes at first follow-up, ${ }^{\mathrm{a}} \mathrm{n}$ (\%) & $43(70)$ & $28(78)$ & 0.368 \\
\hline Resolution of SRF in treatment-naive eyes at final follow-up, ${ }^{\mathrm{b}} \mathrm{n}$ (\%) & $55(87)$ & $35(95)$ & $0.24 I$ \\
\hline Presence of PCRD before initial PDT, $n$ (\%) & $25 / 8 \mid(3 I)$ & $\mathrm{n} / \mathrm{a}^{\mathrm{c}}$ & $\mathrm{n} / \mathrm{a}$ \\
\hline Resolution of PCRD at first follow-up examination, ${ }^{\mathrm{a}} \mathrm{n}(\%)$ & I I/25 (44) & $\mathrm{n} / \mathrm{a}^{\mathrm{c}}$ & $\mathrm{n} / \mathrm{a}$ \\
\hline Resolution of PCRD at final follow-up examination, ${ }^{\mathrm{b}} \mathrm{n}(\%)$ & $13 / 25(52)$ & $\mathrm{n} / \mathrm{a}^{\mathrm{c}}$ & $\mathrm{n} / \mathrm{a}$ \\
\hline
\end{tabular}

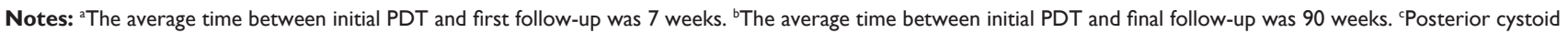
retinal degeneration was only present in the case group. Bold font indicates data were considered statistically significant.

Abbreviations: BCVA, best-corrected visual acuity; CSC, central serous chorioretinopathy; ETDRS, Early Treatment of Diabetic Retinopathy Study; n/a, not applicable; PCRD, posterior cystoid retinal degeneration; PDT, photodynamic therapy; SRF, subretinal fluid. 


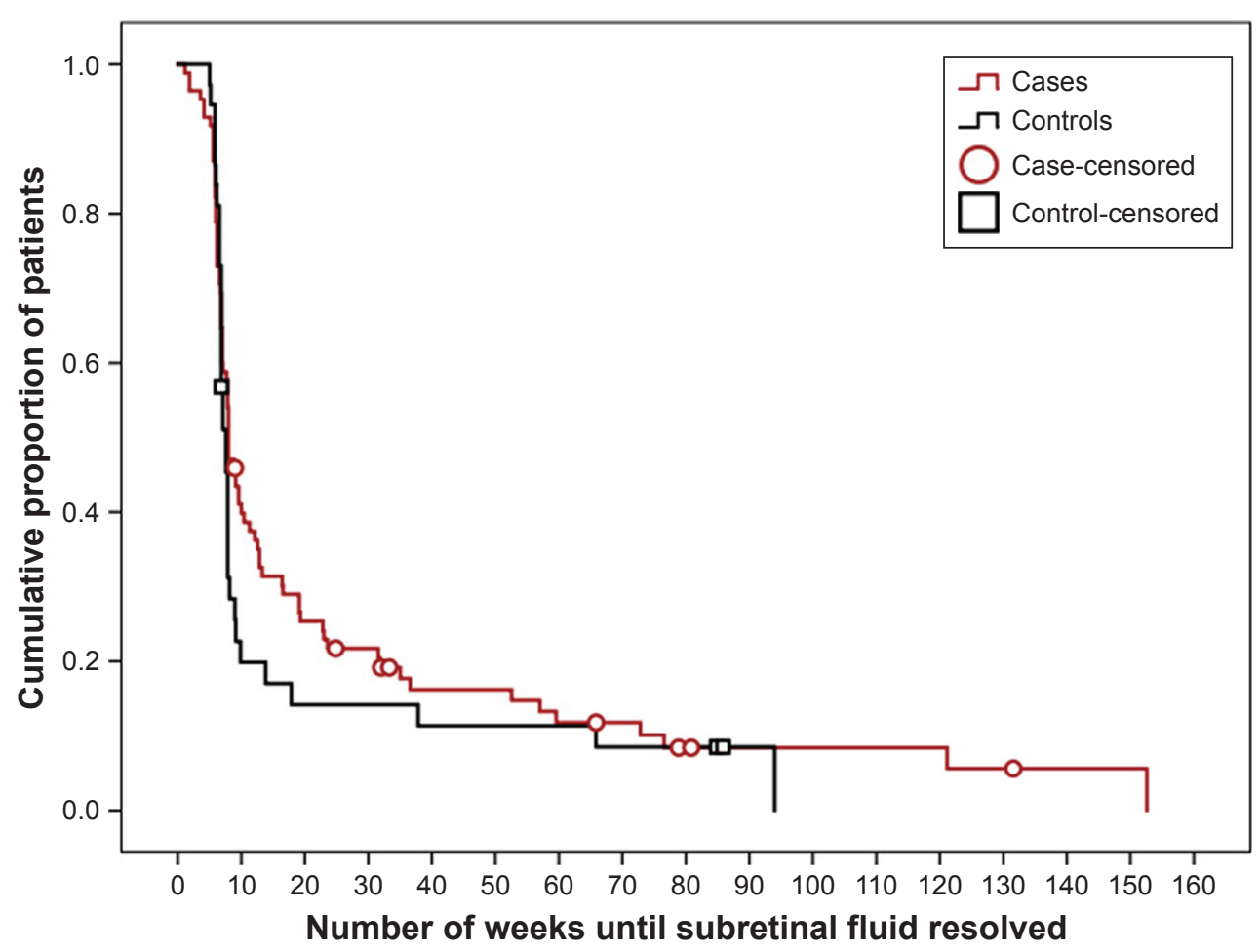

Figure 2 Kaplan-Meier curve showing the cumulative fraction of patients treated for chronic central serous chorioretinopathy (cCSC).

Notes: Endpoint: "Complete resolution of subretinal fluid (SRF) after photodynamic therapy"; the median duration to SRF resolution in cCSC patients with a severe phenotype of the disease (cases) was 8 weeks (95\% Cl: 7-9). In cCSC patients who did not meet the criteria of severity (controls), the median duration was 7 weeks (95\% Cl: 7-8; log-rank test; $P=0.281$ ).

\section{Location of leakage sites in severe cCSC}

The active leakage spots on FA in severe cCSC cases were located inside the largest hyperfluorescent and/or hypofluorescent area of RPE abnormalities in 57 eyes (70\%). In 12 eyes (14\%), the leakage was located on the edges of this largest region of RPE changes, and in 4 eyes (5\%), the leakage came from outside the largest hyperfluorescent and/or hypofluorescent area of RPE changes. In 8 eyes (10\%) of cases, multiple locations of leakage were observed (Figure 4). In 3 cases (4\%) of severe cCSC, hard exudates were seen on fundus photography without evidence of (neo)vascular abnormalities on FA and/or ICGA. These exudates resolved after a single PDT treatment in all cases. Geographic atrophy was not observed in any of the cCSC cases.

\section{Discussion}

In this multicenter study of severe cCSC, PDT with reduced settings showed a similar therapeutic effect compared with a control group of nonsevere cCSC with regard to resolution of SRF. Although visual acuity did increase after PDT, the final outcome was less favorable in the severe cCSC cases. This group also had a worse pretreatment baseline BCVA presumably due to a long-standing active disease. Therefore, an early PDT treatment may reduce the chance of an irreversible decline of visual acuity in severe cCSC.

In the present study, severely affected cCSCs and nonsevere cCSCs showed mostly comparable baseline characteristics before PDT treatment (Table 1). However, in severe cCSC, the mean time from the first CSC diagnosis to the final visit was 4 years longer than in controls. This may reflect the referral pattern in more complex cCSC cases to a tertiary medical center, and the follow-up pattern in these cases. A longer follow-up duration was also described by Otsuka et al in their study on $25 \mathrm{cCSC}$ cases with a severe variant of the disease. The mean follow-up in this study was 10.6 years. ${ }^{25}$

Despite the fact that baseline BCVA was significantly lower in the present severe cCSC cohort compared with the controls, the post-PDT BCVA increased significantly compared with baseline in both cases and controls. However, this improvement was larger in the control group at short- and long-term follow-up. Furthermore, we showed that a better baseline BCVA correlated significantly with a better final visual outcome. We postulate that preexisting photoreceptor and RPE damage in severe cCSC may be the primary cause of suboptimal BCVA improvement after PDT. However, Loo et al reported no association between reduced visual acuity and any degree of macular RPE atrophy, although 

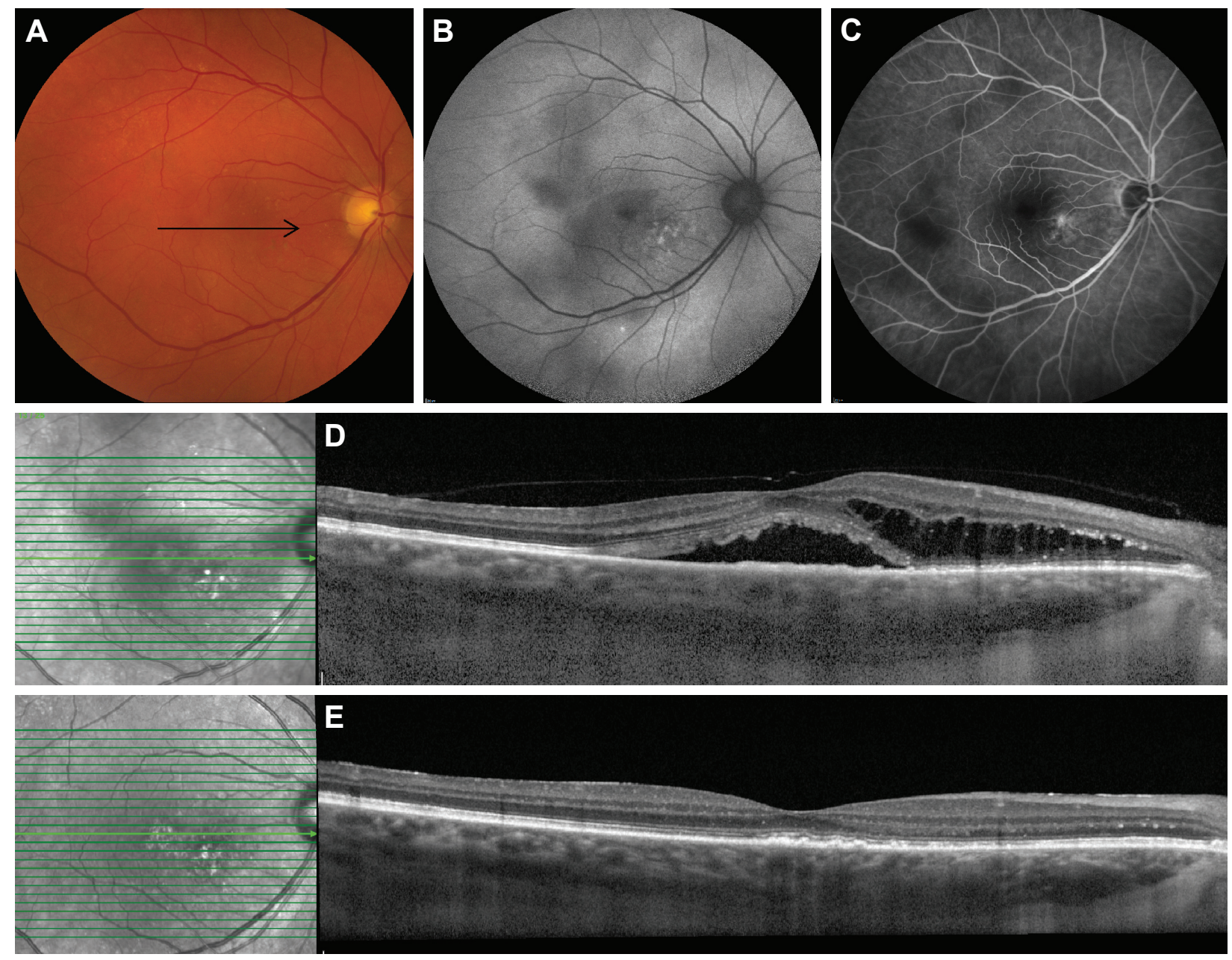

Figure 3 Clinical features on multimodal imaging of the right eye of a 63-year-old female patient with severe chronic central serous chorioretinopathy (A-C). Black arrow on color fundus photography image (A) shows the scanning plane, which is depicted on the SD-OCT scans (D, E). FAF shows multiple speckled hyperautofluorescent changes in the macula together with an irregular surface of hypoautofluorescence, expanding from the fovea to the superior and inferior vascular arcades (B). Fluorescein angiography imaging $(\mathbf{C})$ revealed a limited area of fluorescein leakage with a clear central focus. The areas of hypofluorescence were located more temporal from the fovea and were smaller than on FAF. An SD-OCT scan (D) at first presentation and prior to treatment revealed a subretinal serous fluid accumulation together with a posterior cystoid retinal degeneration in the outer nuclear layer of the retina. At $\sim 2$ months after half-dose photodynamic therapy, both subretinal and intraretinal fluid on OCT had resolved completely $(\mathbf{E})$.

Abbreviations: FAF, fundus autofluorescence imaging; SD-OCT, spectral-domain optical coherence tomography.

they did not specify the surface of the studied RPE atrophy in their cohort. ${ }^{26}$ Balaratnasingam et al described a cohort of 14 patients with a bullous retinal detachment variant of cCSC with diffuse hypo- and hyperautofluorescent retinal abnormalities, descending tracts, and PCRD, which showed similarities with our severe cases, although we did not observe bullous retinal detachments. In contrast to our findings, these authors reported that the mean BCVA at baseline did not differ from final visit BCVA after various treatment strategies. ${ }^{27}$ Future prospective studies should analyze if other parameters besides low baseline BCVA are also predictive for visual outcome, such as microperimetry and chorioretinal imaging characteristics.

In our study, a complete resolution of SRF was achieved in $70 \%$ of severe cases at the first follow-up visit after PDT.
Previous reports have found similar rates of $71 \%-87 \%$, for a general cCSC population. ${ }^{28,29}$ At final follow-up (on average 90 weeks after PDT), a complete resolution of SRF was seen in as much as $88 \%$ of severe cCSC patients. This observation of SRF resolution was comparable to available literatures for a general cCSC population. ${ }^{28-30}$ However, $31 \%$ of our severe cases needed additional treatments, mostly a single additional PDT, due to insufficient treatment effect or SRF recurrence. We found that PDT could be effective even in patients with PCRD. In this study, PCRD resolved completely after initial PDT in $44 \%$ of severe cCSC cases with PCRD. This proportion remained stable until the final follow-up, which is in contrast with findings in previous smaller studies. For instance, Silva et al reported that 10 out of $46 \mathrm{cCSC}$ patients in their study showed PCRD on OCT, and in this study, 

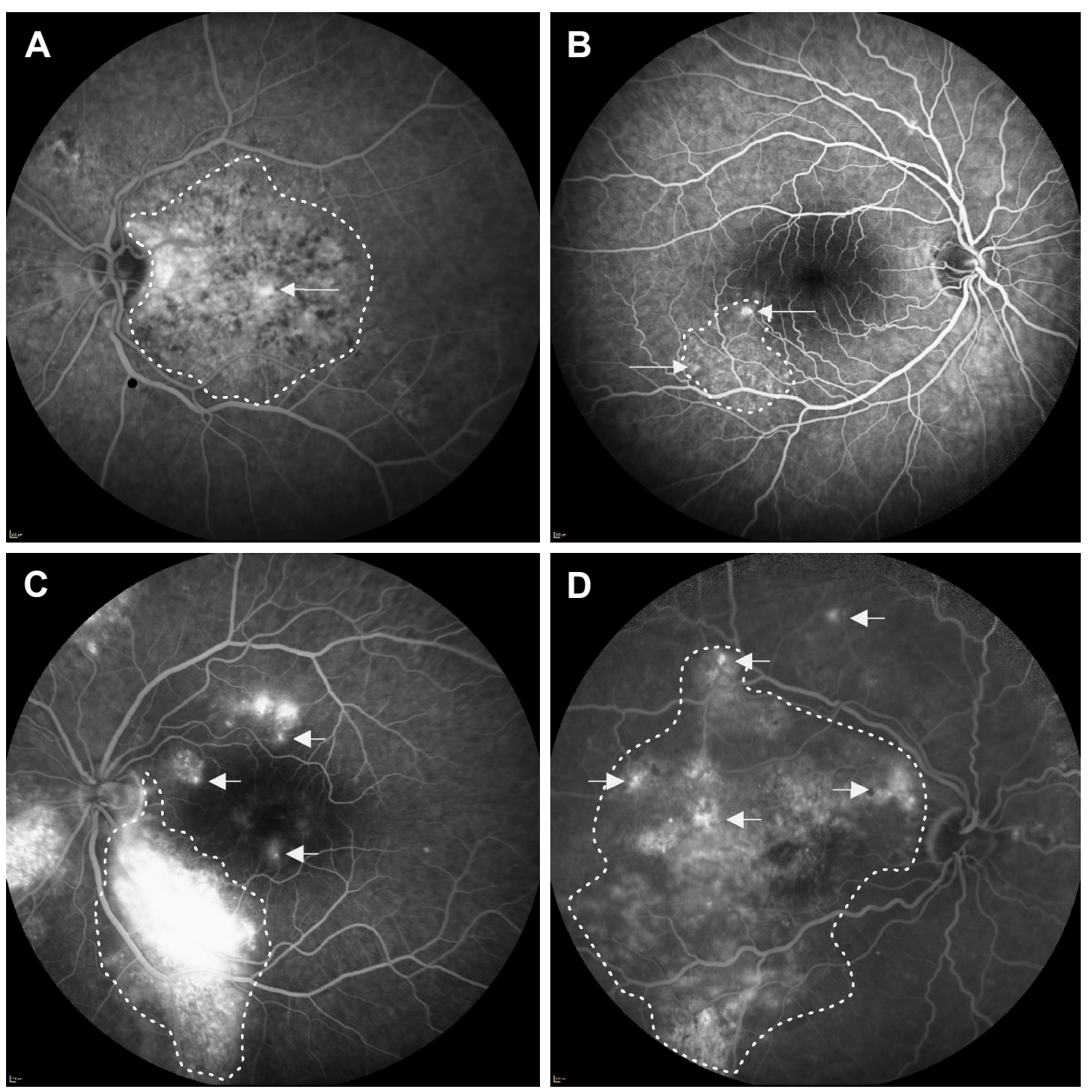

Figure 4 Four categories of eyes with severe chronic central serous chorioretinopathy with active fluorescein leakage on fluorescein angiography.

Notes: This figure illustrates different fluorescein leakage locations (arrows) in relation to the largest area of DARA (dotted line). (A) Category I concerns a leakage point inside the largest area of RPE alterations. (B) Category 2 concerns I or more leakage points located on the edges of the largest region of RPE alterations. (C) In category 3 , leakage points causing macular subretinal fluid are outside the largest zone of RPE alterations. (D) Category 4 concerns cases with multifocal leakage points in several of the aforementioned locations in relation to the area of RPE alterations.

Abbreviations: DARA, diffuse atrophic RPE alterations; RPE, retinal pigment epithelium.

a complete resolution of intraretinal fluid was reported after treatment with full-settings PDT in all of these cases after 4 years of follow-up. ${ }^{31}$ In our study, we did observe a trend of further decrease in number and volume of intraretinal cystoid fluid abnormalities during follow-up after PDT. However, this tendency did not lead to a higher rate of complete PCRD resolution at final follow-up.

The most prominent abnormalities in CSC are choroidal dysfunction, choroidal congestion, and hyperpermeability of the choriocapillaris, which appear to be the primary underlying abnormalities in CSC. The combination of a dysfunctional RPE outer blood-retinal barrier, the insufficient RPE pump function, and a positive pressure gradient from the underlying leaking choroid appears to be a prerequisite for active SRF leakage in CSC. ${ }^{7,32}$ However, it is unclear if the atrophic RPE changes and choroidopathy result from a common pathway. ${ }^{7}$ With regard to the DARA, it is unclear if these changes always result from chronic overlying SRF or if RPE atrophy can also develop directly from an underlying choroidal dysfunction that directly affects the adjacent RPE without the presence of overlying SRF. In our study, DARA areas often did not correspond with the areas of "hot spot(s)" of SRF leakage on FA. We postulate that atrophic RPE changes in severe cCSC may not only occur in areas of prolonged SRF but can also gradually develop primarily as a result of a dysfunctional, leaky underlying pachychoroid. ${ }^{33}$

Although it has been estimated that only $16 \%$ of patients with aCSC may gradually develop a chronic type of disease, ${ }^{34}$ the majority (73\%) of patients that develop a severe phenotype shows signs of chronicity at very first presentation. ${ }^{22}$ Therefore, a timely diagnosis and proper treatment appear crucial, since chronic SRF leakage can 
cause irreversible damage to the neuroretina and RPE, leading to a severe and extensive disease phenotype with a resulting suboptimal vision-related quality of life. ${ }^{5,6,34}$ In the present study, we suggested a definition for severity by taking into account a spectrum of previously described retinal abnormalities, including extensive areas of DARA, multiple foci of recurrent leaking spots, and degenerative intraretinal cystoid abnormalities (PCRD) without evidence of choroidal neovascularization. ${ }^{18,25,35}$ There is currently no consensus on the definition of "extensive" and "large" areas of atrophic RPE abnormalities., ${ }^{2,7}$ Therefore, we somewhat arbitrarily chose a cumulative area of 5 optic disc diameters of atrophic RPE changes in the macula to define DARA. The definition of DARA, and also of multifocal "hot spots", and diffuse leakage must be validated in future studies. Another limitation of the present study is the variety in PDT settings, although half-dose PDT was most frequently used (Table 4). As previous comparative studies have demonstrated that PDT with different reduced settings may be equally effective in treating CSC case, we may assume that the efficacy of PDT was not significantly affected by the specific PDT settings used, but further studies are needed to address this issue more thoroughly. ${ }^{36,37}$

\section{Conclusion}

This study has outlined the clinical spectrum of severe cCSC. It is currently unclear whether aCSC and the spectrum of cCSC manifestations, such as a severe phenotype described in this study, are part of a continuum with the same pathophysiological background, or if they are essentially different diseases. This study shows that patients with severe cCSC respond favorably to PDT with regard to SRF resolution, and that pre-PDT baseline visual acuity is strongly associated with a final BCVA outcome. We, therefore, suggest that an early PDT in patients with severe cCSC phenotypes may improve BCVA more effectively and may prevent further vision loss due to persistent SRF leakage.

\section{Acknowledgments}

This research was supported by the following funding sources: Stichting Leids Oogheelkundig Ondersteuningsfonds, Rotterdamse Stichting Blindenbelangen, Stichting Wetenschappelijk Onderzoek Het Oogziekenhuis, Macula Fonds, Landelijke Stichting voor Blinden en Slechtzienden, Retina the Netherlands, and BlindenPenning. CJFB was supported by a Gisela Thier Fellowship from Leiden University and a ZonMw Veni grant from the Netherlands Organization for Scientific Research (NWO). These sponsors and funding organizations played no role in the design or conduct of this research. An abstract version of this manuscript was presented at the 2016 annual meeting of the Association for Research in Vision and Ophthalmology.

\section{Disclosure}

The authors report no conflicts of interest in this work.

\section{References}

1. Gass JD. Pathogenesis of disciform detachment of the neuroepithelium. Am J Ophthalmol. 1967;63(3):1-139.

2. Nicholson B, Noble J, Forooghian F, Meyerle C. Central serous chorioretinopathy: update on pathophysiology and treatment. Surv Ophthalmol. 2013;58(2):103-126.

3. Liew G, Quin G, Gillies M, Fraser-Bell S. Central serous chorioretinopathy: a review of epidemiology and pathophysiology. Clin Exp Ophthalmol. 2013;41(2):201-214.

4. Fujimoto H, Gomi F, Wakabayashi T, et al. Morphologic changes in acute central serous chorioretinopathy evaluated by fourier-domain optical coherence tomography. Ophthalmology. 2008;115(9):1494-1500, 1500.e1-2

5. Breukink MB, Dingemans AJ, den Hollander AI, et al. Chronic central serous chorioretinopathy: long-term follow-up and vision-related quality of life. Clin Ophthalmol. 2017;11:39-46.

6. Wang M, Munch IC, Hasler PW, Prünte C, Larsen M. Central serous chorioretinopathy. Acta Ophthalmol. 2008;86(2):126-145.

7. Daruich A, Matet A, Dirani A, et al. Central serous chorioretinopathy: Recent findings and new physiopathology hypothesis. Prog Retin Eye Res. 2015;48:82-118.

8. Quin G, Liew G, Ho IV, Gillies M, Fraser-Bell S. Diagnosis and interventions for central serous chorioretinopathy: review and update. Clin Exp Ophthalmol. 2013;41(2):187-200.

9. Gemenetzi M, de Salvo G, Lotery AJ. Central serous chorioretinopathy: an update on pathogenesis and treatment. Eye. 2010;24(12): 1743-1756.

10. Yannuzzi LA. Type A behavior and central serous chorioretinopathy. Trans Am Ophthalmol Soc. 1986;84:799-845.

11. Haimovici R, Koh S, Gagnon DR, et al. Risk factors for central serous chorioretinopathy: a case-control study. Ophthalmology. 2004; 111(2):244-249.

12. de Jong EK, Breukink MB, Schellevis RL, et al. Chronic central serous chorioretinopathy is associated with genetic variants implicated in agerelated macular degeneration. Ophthalmology. 2015;122(3):562-570.

13. Miki A, Kondo N, Yanagisawa S, et al. Common variants in the complement factor $\mathrm{H}$ gene confer genetic susceptibility to central serous chorioretinopathy. Ophthalmology. 2014;121(5):1067-1072.

14. Schubert C, Pryds A, Zeng S, et al. Cadherin 5 is regulated by corticosteroids and associated with central serous chorioretinopathy Hum Mutat. 2014;35(7):859-867.

15. Breukink MB, Schellevis RL, Boon CJ, et al. Genomic copy number variations of the complement component $\mathrm{C} 4 \mathrm{~B}$ gene are associated with chronic central serous chorioretinopathy. Invest Ophthalmol Vis Sci. 2015;56(9):5608-5613.

16. Salehi M, Wenick AS, Law HA, Evans JR, Gehlbach P. Interventions for central serous chorioretinopathy: a network meta-analysis. Cochrane Database Syst Rev. 2015;22(12):CD011841.

17. van Dijk EHC, Fauser S, Breukink MB, et al. Half-Dose Photodynamic Therapy versus High-Density Subthreshold Micropulse Laser Treatment in Patients with Chronic Central Serous Chorioretinopathy: The PLACE Trial. Ophthalmology. 2018;125(10):1547-1555.

18. Piccolino FC, de La Longrais RR, Manea M, Cicinelli S. Posterior cystoid retinal degeneration in central serous chorioretinopathy. Retina. 2008;28(7):1008-1012. 
19. Iida T, Yannuzzi LA, Spaide RF, et al. Cystoid macular degeneration in chronic central serous chorioretinopathy. Retina. 2003;23(1): 137-138.

20. von Winning CH, Oosterhuis JA, Renger-van Dijk AH, HornstraLimburg H, Polak BC. Diffuse retinal pigment epitheliopathy. Ophthalmologica. 1982;185(1):7-14.

21. Yannuzzi LA, Slakter JS, Kaufman SR, Gupta K. Laser treatment of diffuse retinal pigment epitheliopathy. Eur J Ophthalmol. 1992;2(3): 103-114.

22. Mohabati D, van Rijssen TJ, van Dijk EH, et al. Clinical characteristics and long-term visual outcome of severe phenotypes of chronic central serous chorioretinopathy. Clin Ophthalmol. 2018;12:1061-1070.

23. Polak BC, Baarsma GS, Snyers B. Diffuse retinal pigment epitheliopathy complicating systemic corticosteroid treatment. Br J Ophthalmol. 1995;79(10):922-925.

24. Gregori NZ, Feuer W, Rosenfeld PJ. Novel method for analyzing snellen visual acuity measurements. Retina. 2010;30(7):1046-1050.

25. Otsuka S, Ohba N, Nakao K. A long-term follow-up study of severe variant of central serous chorioretinopathy. Retina. 2002;22(1):25-32.

26. Loo RH, Scott IU, Flynn HW, et al. Factors associated with reduced visual acuity during long-term follow-up of patients with idiopathic central serous chorioretinopathy. Retina. 2002;22(1):19-24.

27. Balaratnasingam C, Freund KB, Tan AM, et al. Bullous variant of central serous chorioretinopathy: expansion of phenotypic features using multimethod imaging. Ophthalmology. 2016;123(7):1541-1552.

28. Fujita K, Imamura Y, Shinoda K, et al. One-year outcomes with halfdose verteporfin photodynamic therapy for chronic central serous chorioretinopathy. Ophthalmology. 2015;122(3):555-561.
29. Lim JI, Glassman AR, Aiello LP, et al. Collaborative retrospective macula society study of photodynamic therapy for chronic central serous chorioretinopathy. Ophthalmology. 2014;121(5):1073-1078.

30. Nicoló M, Eandi CM, Alovisi C, et al. Half-fluence versus half-dose photodynamic therapy in chronic central serous chorioretinopathy. Am J Ophthalmol. 2014;157(5):1033-1037.

31. Silva RM, Ruiz-Moreno JM, Gomez-Ulla F, et al. Photodynamic therapy for chronic central serous chorioretinopathy: a 4-year follow-up study. Retina. 2013;33(2):309-315.

32. Agrawal R, Chhablani J, Tan KA, Shah S, Sarvaiya C, Banker A. Choroidal vascularity index in central serous chorioretinopathy. Retina. 2016;36(9):1646-1651.

33. Warrow DJ, Hoang QV, Freund KB. Pachychoroid pigment epitheliopathy. Retina. 2013;33(8):1659-1672.

34. Castro-Correia J, Coutinho MF, Rosas V, Maia J. Long-term follow-up of central serous retinopathy in 150 patients. Doc Ophthalmol. 1992; 81(4):379-386.

35. Yannuzzi LA, Shakin JL, Fisher YL, Altomonte MA. Peripheral retinal detachments and retinal pigment epithelial atrophic tracts secondary to central serous pigment epitheliopathy. Ophthalmology. 1984;91(12): 1554-1572.

36. Kim YK, Ryoo NK, Woo SJ, Park KH. Comparison of visual and anatomical outcomes of half-fluence and half-dose photodynamic therapy in eyes with chronic central serous chorioretinopathy. Graefes Arch Clin Exp Ophthalmol. 2015;253(12):2063-2073.

37. Alkin Z, Perente I, Ozkaya A, et al. Comparison of efficacy between low-fluence and half-dose verteporfin photodynamic therapy for chronic central serous chorioretinopathy. Clin Ophthalmol. 2014;8:685-690.
Clinical Ophthalmology

\section{Publish your work in this journal}

Clinical Ophthalmology is an international, peer-reviewed journal covering all subspecialties within ophthalmology. Key topics include: Optometry; Visual science; Pharmacology and drug therapy in eye diseases; Basic Sciences; Primary and Secondary eye care; Patient Safety and Quality of Care Improvements. This journal is indexed on Submit your manuscript here: http://www.dovepress.com/clinical-ophthalmology-journal

\section{Dovepress}

PubMed Central and CAS, and is the official journal of The Society of Clinical Ophthalmology (SCO). The manuscript management system is completely online and includes a very quick and fair peer-review system, which is all easy to use. Visit http://www.dovepress.com/ testimonials.php to read real quotes from published authors. 